\title{
Vom Arbeitsmarkt in den Ruhestand: Die Einkommen deutscher Rentner und Rentnerinnen
}

\author{
Anette Reil-Held und Reinhold Schnabel \\ Fakultät für Volkswirtschaftslehre und Sonderforschungsbereich 504 \\ Universität Mannheim
}

SFB 504 Discussion Paper

\section{Zusammenfassung}

Dieser Beitrag behandelt den Übergang vom Arbeitsmarkt in den Ruhestand und die Einkommenslage von Rentnerhaushalten in Deutschland. Wir stützen unsere empirische Analyse auf das Sozio-ökonomische Panel, die Einkommens- und Verbrauchsstichprobe 1993 und Daten der gesetzlichen Rentenversicherung.

In einer empirische Untersuchung von Rentenzugang und Erwerbsbeteiligung älterer Menschen zeigen wir, wie sehr Ruhestand und Rentenbezug auseinanderfallen. Zum einen erfolgt oftmals ein Ausstieg aus dem Erwerbsleben, lange bevor eine Rente bezogen wird. Zum anderen ist die Phase des Rentenbezugs sehr häufig mit weiterer Erwerbstätigkeit verbunden, was sich in Entwicklung und Zusammensetzung der Rentnereinkommen widerspiegelt. Die Einkommenslage des überwiegenden Teils der Rentnerhaushalte ist gesichert: Armut ist heute kein spezifisches Problem der Älteren mehr, wie sich auch an dem Sozialhilfebezug erkennen läßt. Für Personen mit geringen Arbeitseinkommen oder kurzen Erwerbshistorien können sich jedoch Renteneinkommen ergeben, die unter dem Sozialhilfeniveau liegen. Betroffen hiervon sind in erster Linie Frauen. In der Zukunft wird sich die relative Lage von älteren Frauen durch die gestiegene Erwerbsbeteiligung weiter verbessern. Die aufgrund der demographischen Entwicklung zu erwartende Senkung der Ersatzquote wird zwar die relative Einkommensposition von Rentnerhaushalten schwächen, doch könnte dies durch private Ersparnis, erhöhte Erwerbsbeteiligung und spätere Verrentung kompensiert werden.

Dieser Beitrag ist im Rahmen des Projektes B1 des Sonderforschungsbereichs 504 am Lehrstuhl für Makroökonomik und Wirtschaftspolitik entstanden. Teile des Beitrages beruhen auf gemeinsamen Arbeiten mit Axel Börsch-Supan, dem wir zu großem Dank verpflichtet sind. Wir danken der Deutschen Forschungsgemeinschaft, Sonderforschungsbereich 504 an der Universität Mannheim für finanzielle Unterstützung.

Anette Reil-Held, Fakultät für Volkswirtschaftslehre, Universität Mannheim, D-68131 Mannheim Email: anette@econ.uni-mannheim.de

Reinhold Schnabel, Fakultät für Volkswirtschaftslehre, Universität Mannheim, D-68131 Mannheim

Email: reinhold@econ.uni-mannheim.de 


\section{Einführung}

Dieser Beitrag behandelt den Übergang vom Arbeitsmarkt in den Ruhestand und die Einkommenslage von Rentnerhaushalten in Deutschland. Wir stützen unsere empirische Analyse auf das Sozio-ökonomische Panel, die Einkommens- und Verbrauchsstichprobe 1993 und Daten der gesetzlichen Rentenversicherung.

Zunächst erfolgt eine empirische Untersuchung des Rentenzugangs und der Erwerbsbeteiligung älterer Menschen. In unserer empirischen Analyse zeigen wir auch, wie sehr Ruhestand und Rentenbezug auseinanderfallen. Erstens kann der Übergang in den Ruhestand direkt vom Erwerbsleben aus erfolgen oder indirekt über Zwischenstationen wie den betrieblichen Vorruhestand. Zweitens ist die Phase des Rentenbezugs sehr häufig keine inaktive Phase, denn zahlreiche - nicht nur jüngere - Rentner gehen weiterhin einer Erwerbstätigkeit nach. Dieser allmähliche Rückzug aus dem Erwerbsleben spiegelt sich in der Entwicklung und der Zusammensetzung der Einkommen von Rentnerhaushalten wider.

Der zweite Abschnitt widmet sich dem Niveau und der Verteilung der Einkommen von Rentnerhaushalten. Die wirtschaftliche Lage deutscher Rentner und Rentnerinnen kann sowohl im internationalen Vergleich als auch im Vergleich mit der erwerbsfähigen Bevölkerung als gesichert bezeichnet werden. Dies liegt insbesondere an der gesetzlichen Rentenversicherung. Aufgrund der Beitragsbezogenheit der Renten können sich aber für Personen mit geringen Arbeitseinkommen oder kurzen Erwerbshistorien Einkommen ergeben, die deutlich unter dem Sozialhilfeniveau liegen. Betroffen hiervon sind in erster Linie Frauen mit längeren Erwerbsunterbrechungen. Jedoch ist Armut heute kein spezifisches Problem der Älteren mehr, wie sich an dem Sozialhilfebezug erkennen läßt.

Abschließend gehen wir der Frage nach, welchen Einflüssen die Einkommenslage von Rentnerhaushalten aufgrund der Entwicklung von Demographie und Erwerbsverhalten ausgesetzt wird. Wir zeigen, daß sich die relative Lage von älteren Frauen durch die absehbare, zunehmende Erwerbsbeteiligung sogar weiter verbessern wird. Die aufgrund der demographischen Entwicklung zu erwartende Senkung der Nettoersatzquote ${ }^{1}$ vermindert zwar die relative Einkommensposition von Rentnerhaushalten, doch kann dies zum großen Teil kompensiert werden durch private Ersparnis, erhöhte Erwerbsbeteiligung und reduzierte Frühverrentung.

\footnotetext{
${ }^{1}$ Im folgenden ist hiermit immer der Quotient aus Nettostandardrente und durchschnittlichem Nettoarbeitsentgelt gemeint.
} 


\section{Vom Arbeitsmarkt in den Ruhestand}

Der Übergang zwischen „drinnen und draußen“, vom Arbeitsmarkt in den Ruhestand, vollzieht sich in den meisten Fällen bereits vor dem offiziellen Regelrentenalter von 65 Jahren. Dies spiegelt sich in einem durchschnittlichen Rentenzugangsalter von nur 60 Jahren in den alten und 58 Jahren in den neuen Bundesländern wider (VDR 1997). Der Rückzug aus dem Erwerbsleben vollzieht sich dabei sehr häufig nochmals einige Jahre früher, also vor dem ersten Rentenbezug, wobei reguläre oder betriebliche Vorruhestandsprogramme und die Arbeitslosenversicherung beansprucht werden. Nach der Verrentung beziehen „Ruheständler“ neben ihrer Rente häufig noch zusätzliche Erwerbseinkommen. ${ }^{2}$ Man kann daher in vielen Fällen von einem gleitenden Übergang in den Ruhestand sprechen.

\section{Rentenberechtigung und Rentenalter}

Der Übergang vom Arbeitsmarkt in den Ruhestand wird sehr stark durch institutionelle Regeln geprägt, die den Rentenanspruch bestimmen. Wir können uns hierbei auf die Regelungen der Rentenreform 1972 beschränken, da sich unsere empirische Analyse auf die Jahre vor 1996 bezieht und die alten Regelungen noch für die bisherigen Rentenzugänge gelten. ${ }^{3}$

Im Rahmen der Versichertenrenten sind zunächst Altersrenten und Renten wegen verminderter Erwerbsfähigkeit zu unterscheiden. Während es für Renten wegen Erwerbsminderung keine Altersgrenze gibt, können Altersrenten frühestens im Alter von 60 Jahren beansprucht werden. Es gibt fünf Arten der Altersrenten. Die „Regelaltersrente“ kann ab dem fünfundsechzigsten Lebensjahr in Anspruch genommen werden und setzt nur fünf Versicherungsjahre voraus. Die „Altersrente für langjährig Versicherte“ kann bereits im Alter von 63 beansprucht werden, sofern mindestens 35 Versicherungsjahre vorgewiesen werden. Die Einführung dieser, auch als „flexible Altersrente“ bezeichneten Rente, war ein Hauptelement der Rentenreform von 1972 und hat in den Folgejahren die Frühverrentung deutlich ansteigen lassen (Börsch-Supan und Schnabel 1998). Die übrigen drei Rentenarten ermöglichen bereits eine Verrentung mit 60 Jahren. Es sind dies die „Altersrente für Frauen“, die „Altersrente wegen Arbeitslosigkeit“ und die „Altersrente für Schwerbehinderte, Berufs- und Erwerbsunfähi$g e$ “. Letztere erfordert mindestens 35 Versicherungsjahre. Für die Rente wegen Arbeitslosig-

\footnotetext{
${ }^{2}$ Dies ist vom Gesetzgeber - etwa im Falle der Berufsunfähigkeit - so vorgesehen.

${ }^{3}$ Siehe Börsch-Supan und Schnabel (1998) für eine detaillierte Beschreibung der gesetzlichen Rentenversicherung. Hinterbliebenenrenten werden hier zunächst ausgeklammert.
} 
keit sind nur 15 Versicherungsjahre erforderlich, jedoch müssen die letzten zehn Jahre vor dem Rentenantrag mit mindestens 8 Pflichtbeitragsjahren belegt sein. ${ }^{4}$ Frauen müssen für die Rente ab 60 nur 15 Versicherungsjahre aufweisen, von denen 10 nach dem 40. Lebensjahr liegen müssen.

\section{Abbildung 1: Verteilung des Rentenzugangs nach Alter und Geschlecht im Jahr 1995}
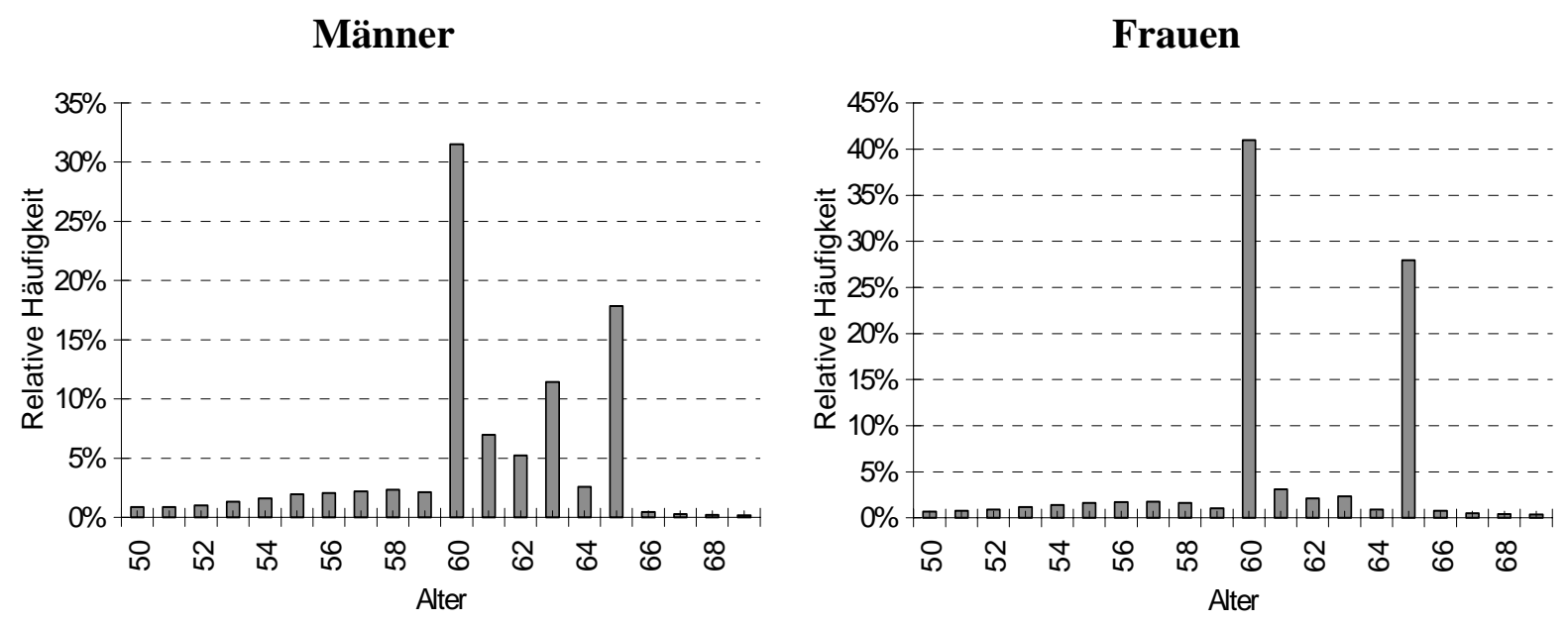

Anmerkungen: Verteilung des Alters von Versicherten, die 1995 zum ersten Mal eine Rentenzahlung der Gesetzlichen Rentenversicherung erhielten. Quelle: VDR, 1997, Rentenzugang 1995.

Wie deutlich sich die gesetzlichen Regelungen in der Verteilung des Rentenzugangsalters widerspiegeln, zeigt Abbildung 1. Das Rentenzugangsalter ist hier definiert als das Alter, in dem erstmals eine gesetzliche Rente bezogen wird. Bei Männer häufen sich die Rentenzugänge im Alter von 60, 63 und 65. Im Jahr 1995 war das Alter 60 das weitaus häufigste Eintrittsalter, gefolgt von dem Alter 65 und 63. Nach dem Alter von 65 geht kaum jemand in Rente. Die Rentenzugänge vor dem Alter 60 addieren sich immerhin zu beachtlichen 27,6 Prozent in den alten Bundesländern und 22,33 Prozent in Deutschland. ${ }^{5}$ Bei den Rentenzugängen von Frauen gibt es dagegen im Alter von 63 keine Spitze, da Frauen bereits mit 60 eine vorgezogene Altersrente beziehen können, sofern sie die Anforderung an die Beitragszeiten erfüllen. Frauen mit kurzen Erwerbshistorien können erst zum 65. Lebensjahr in Rente gehen. Auch bei Frauen spielt die Verrentung vor dem sechzigsten Geburtstag eine große Rolle mit einem Anteil von 18,5 Prozent im Jahr 1995 (VDR 1997).

${ }^{4}$ Die Anforderungen an die Dauer der Arbeitslosigkeit wurden mehrfach geändert. Zuletzt mußten in den anderthalb Jahren vor dem Rentenantrag mindestens 52 Wochen Arbeitslosigkeit nachgewiesen werden.

${ }^{5}$ Diese Zahlen liegen etwas niedriger als die Rentenzugänge wegen verminderter Erwerbsfähigkeit, da diese in einigen Fällen auch nach dem 60. Geburtstag vorkommen. 
Abbildung 2 zeigt anhand einer Auswertung des SOEP, wie sich das Rentenzugangsverhalten auf den Bestand an Rentnern und Rentnerinnen auswirkt. Im Alter von 59 bezogen bereits 23,5 Prozent der Männer und 18,5 Prozent der Frauen eine eigene Rente; im Alter von 60 waren es bereits 44,5 respektive 52,0 Prozent. Ab dem Alter von 67 beziehen nahezu alle Männer eine eigene Rente, jedoch nur etwa 90 Prozent der Frauen.

\section{Abbildung 2: Rentenbezug nach Alter und Geschlecht}

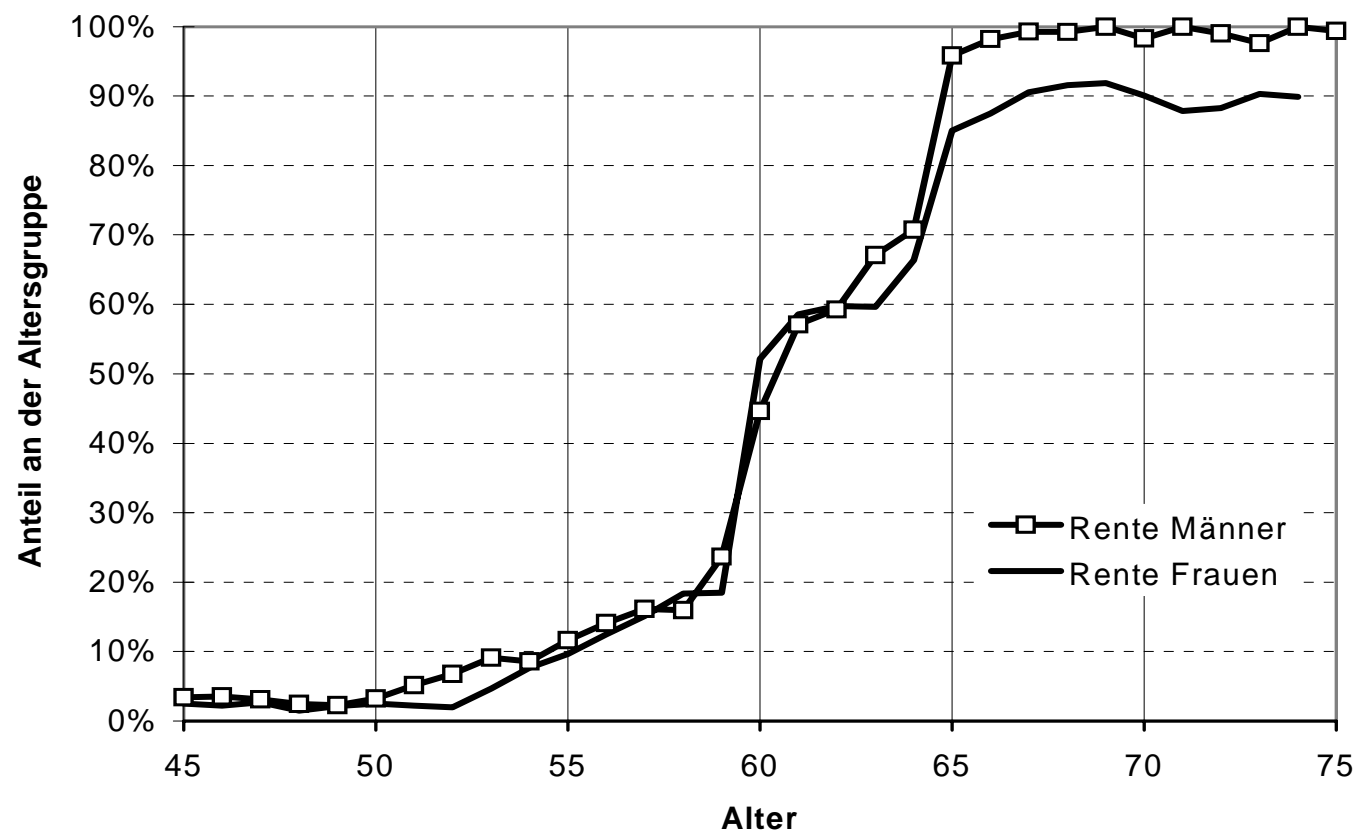

Anmerkungen: Rente = Empfänger von Pensionen, Alters- oder Invaliditätsrenten als Anteil an den Befragungspersonen der Altersgruppe. Ohne Hinterbliebenenrenten.

Quelle: Eigene Berechnungen auf Basis des SOEP 1993-95, neue und alte Bundesländer.

\section{Entwicklung der altersspezifischen Erwerbsbeteiligung}

Das niedrige Durchschnittsrentenalter korrespondiert mit einer niedrigen Erwerbsbeteiligung im Alter. Die Erwerbsbeteiligung nimmt etwa in der Altersgruppe der 40-Jährigen ein Maximum an und geht bereits ab einem Alter von 50 Jahren deutlich zurück. So waren im Jahr 1990 nur noch 35 Prozent der 60- bis 64-Jährigen Männer erwerbstätig. Die Erwerbsbeteiligung älterer Menschen in Deutschland erfuhr - wie in den meisten Industrienationen - im langfristigen Vergleich einen drastischen Rückgang. Dieser beschleunigte sich seit 1970 (vgl. Tabelle 1), was teilweise auf die 1972 eingeführte flexible Altersgrenze zurückzuführen ist. Die Erwerbsbeteiligung der Männer ging bei allen über 50-Jährigen zwischen 1970 und 1990 zurück. Im Gegensatz dazu stieg die Erwerbsbeteiligung der Frauen in allen Altersgruppen unter 60 an, was auf die allgemein steigende Arbeitsmarktbeteiligung von Frauen zurückzu- 
führen ist. Der Anstieg für die 50-59jährigen Frauen ist besonders erwähnenswert, weil er im Kontrast zur sinkenden Erwerbstätigkeit der Männer in diesem Alter steht.

Tabelle 1: Erwerbsquoten der Frauen und Männer in den alten Bundesländern

\begin{tabular}{|c|c|c|c|c|c|c|c|c|}
\hline \multirow[b]{2}{*}{ Alter } & \multicolumn{4}{|c|}{ Frauen } & \multicolumn{4}{|c|}{ Männer } \\
\hline & 1960 & 1970 & 1980 & 1990 & 1960 & 1970 & 1980 & 1990 \\
\hline $45-49$ & 40.9 & 48.9 & 52.2 & 66.7 & $96.1 \%$ & 96.8 & 96.8 & 96.5 \\
\hline $50-54$ & 37.4 & 44.8 & 47.1 & 57.8 & 94.1 & 95.1 & 93.3 & 93.2 \\
\hline $55-59$ & 32.4 & 37.2 & 38.7 & 43.8 & 89.0 & 89.2 & 82.3 & 81.1 \\
\hline $60-64$ & 20.9 & 22.5 & 13.0 & 12.5 & 72.5 & 74.7 & 44.2 & 35.0 \\
\hline $65+$ & 8.2 & 6.5 & 3.0 & 2.0 & 22.6 & 19.7 & 7.4 & 5.3 \\
\hline
\end{tabular}

Quelle: Börsch-Supan und Schnabel, 1998b, basierend auf dem Mikrozensus.

\section{Vorruhestand}

Ein Ausstieg aus der Erwerbstätigkeit vor dem 60. Lebensjahr kann nicht nur über eine Rente wegen verminderter Erwerbsfähigkeit erfolgen, sondern auch durch eine Variante des regulären oder informellen Vorruhestands. Eine Kombination aus Arbeitslosenunterstützung und betrieblichen Vorruhestandsprogrammen ermöglicht einen Rückzug aus dem Erwerbsleben vor dem sechzigsten Geburtstag. In einer häufig gewählten Variante vereinbaren Arbeitgeber und Arbeitnehmer eine Auflösung des Arbeitsverhältnisses, die mit einer einmaligen Abfindung oder einer laufenden Zahlung verbunden ist. Nach der Entlassung können ältere Arbeitnehmer zumindest drei Jahre Arbeitslosenunterstützung beziehen und mit Vollendung des sechzigsten Lebensjahres Altersrente wegen Arbeitslosigkeit erhalten.

Wie stark der Rückzug vom Arbeitsmarkt und der Bezug von Rente auseinanderfallen, macht Abbildung 3 deutlich. Die Bezeichnung „Ruhestand” umfaßt darin alle Personen, die sich selbst als im Ruhestand befindlich bezeichnen, unabhängig davon, ob sie eine Rente beziehen. Etwa 40 Prozent aller westdeutschen Männer im Alter von 57 bis 59 Jahren stuften sich selbst ein als „im Ruhestand” befindlich. Doch nur die Hälfte dieser Gruppe bezog eine Erwerbsoder Berufsunfähigkeitsrente, die andere Hälfte bezog eine Form von betrieblichen Vorruhestandszahlungen oftmals in Kombination mit Arbeitslosenunterstützung (siehe auch BörschSupan und Schnabel 1998). Dieser „Vorruhestand“ ist weit verbreitet: der Gipfel liegt im Bereich der Altersgruppe 57-59 bei 20 Prozent für Männer und 25 Prozent für Frauen. Im Alter von 65 erhalten dann schließlich nahezu alle Männer und etwa 90 Prozent der Frauen eine eigene Rente aus der gesetzlichen Rentenversicherung. 
(a) Männer

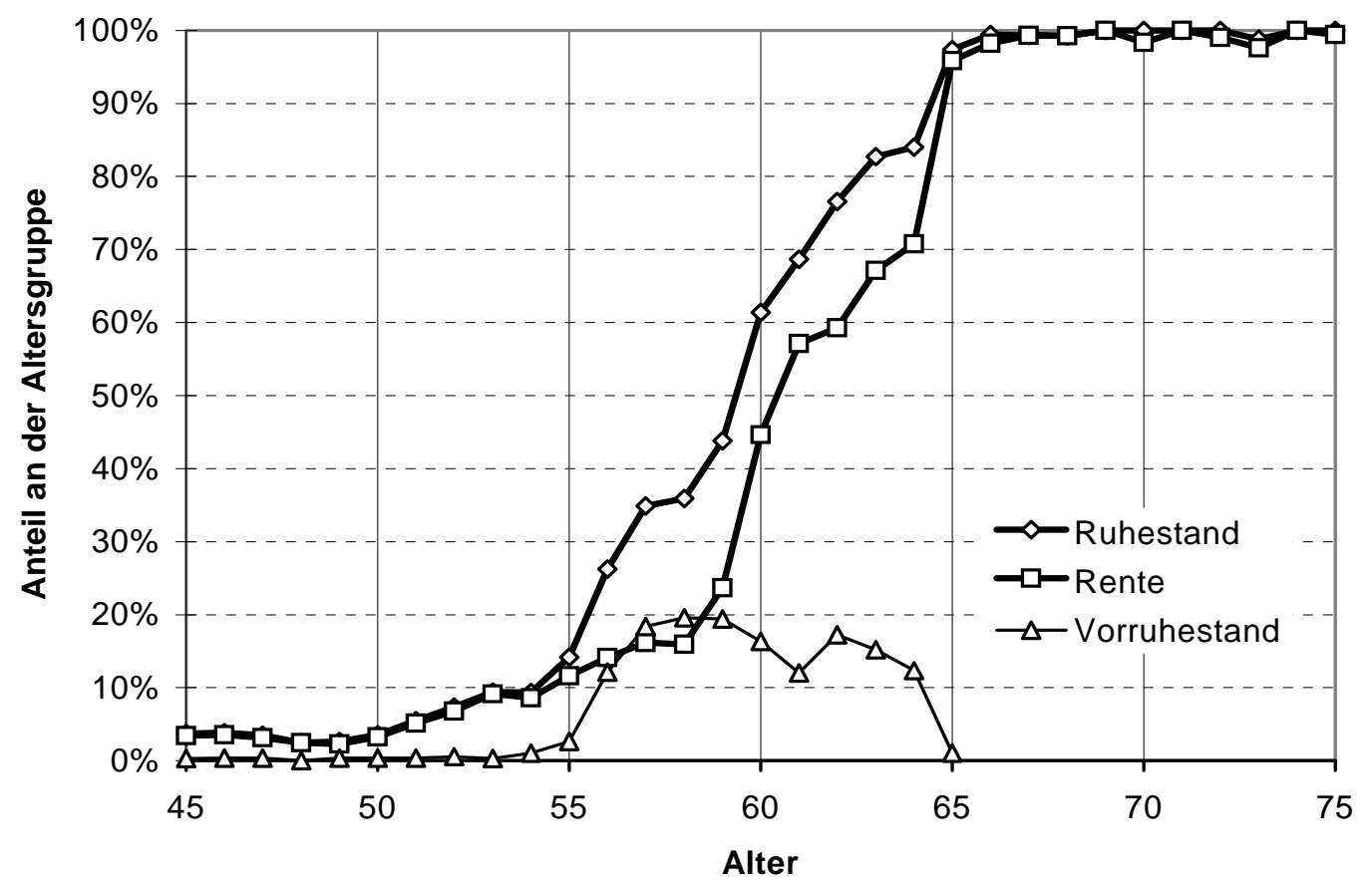

(b) Frauen

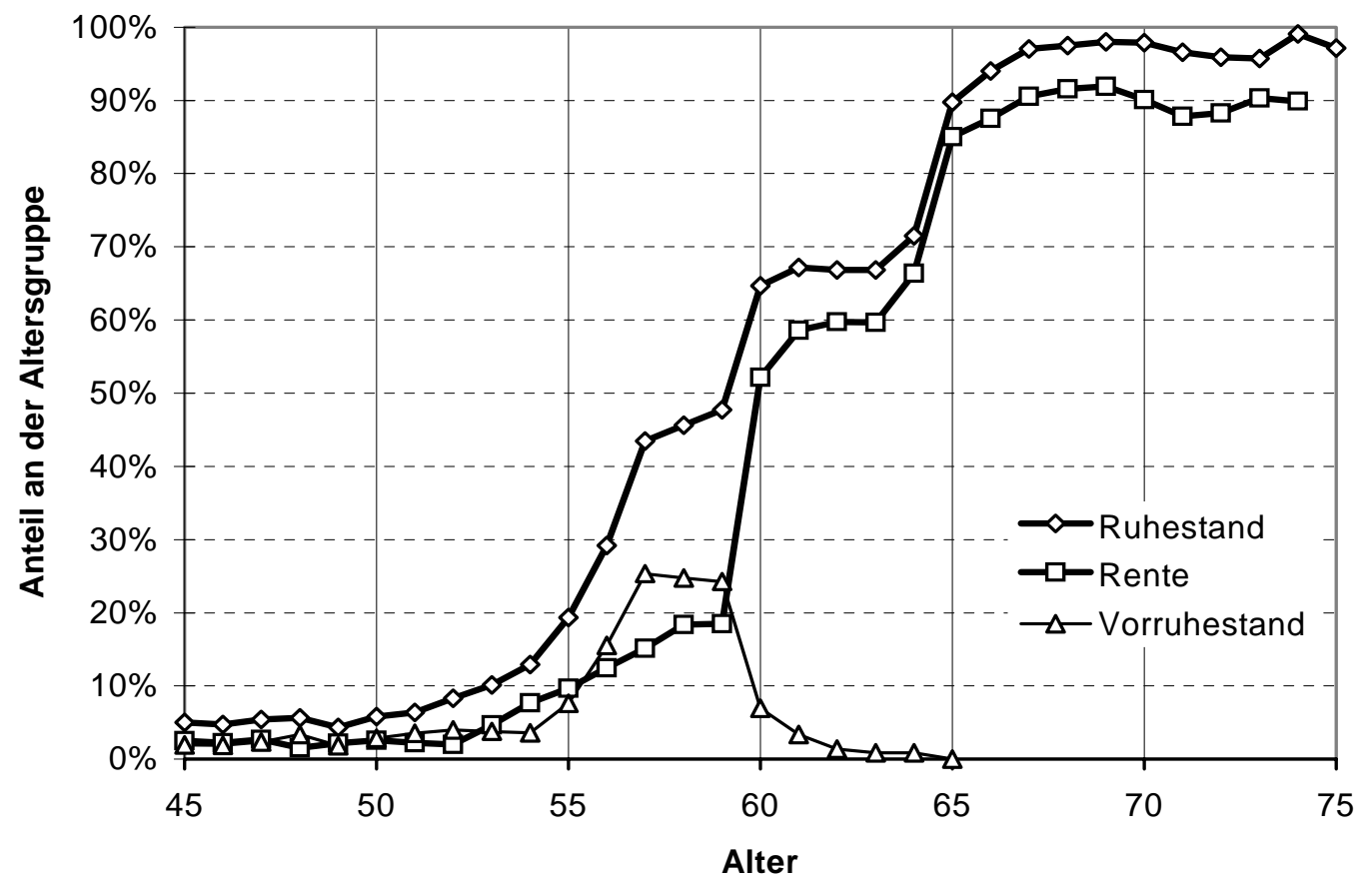

Anmerkungen: Ruhestand = Personen, die sich selbst als im Ruhestand befindlich bezeichnen; Rente = Empfänger von Pensionen, Alters- oder Invaliditätsrenten; Vorruhestand = Personen im Ruhestand, die Bezüge vom Arbeitgeber und/oder Arbeitslosenunterstützung beziehen und nicht erwerbstätig sind. Jeweils als Anteil an den Befragungspersonen im jeweiligen Alter.

Quelle: Börsch-Supan und Schnabel, 1998b, basierend auf dem SOEP 1993-95, neue und alte Bundesländer. 
Es zeigt sich also, daß nach dem Alter 55 eine erhebliche Zahl der Erwerbspersonen aus dem Erwerbsleben ausscheidet, ohne sofort eine gesetzliche Rente zu beziehen. Würde nur der Rentenbezug betrachtet, dann ergäbe sich eine beträchtliche Unterschätzung des Ausmaßes des frühzeitigen Rückzugs vom Arbeitsmarkt.

\section{Erwerbstätigkeit in der Rentephase}

Zwar sind bis zum Alter von 65 Jahren nahezu alle Personen in Rente gegangen, doch ist das nicht gleichbedeutend mit einem vollständigen Rückzug vom Arbeitsmarkt. In aller Regel wird nur das, was man primäre Erwerbstätigkeit nennen könnte, aufgegeben. Sehr häufig schließt sich daran eine Phase der Nebenerwerbstätigkeit an. Diese Phase endet für viele Ruheständler erst jenseits des Alters von 75. Diese Tatsache wird durch die offiziellen Zahlen des statistischen Bundesamtes, die auf dem Mikrozensus und damit auf dem Konzept der Berichtswoche beruhen, verschleiert.

Tabelle 2: Anteil der Nukleus-Haushalte mit Einkünften aus Erwerbstätigkeit

\begin{tabular}{lcccc}
\hline & $\mathbf{6 0 - 6 4}$ & $\mathbf{6 5 - 6 9}$ & $\mathbf{7 0 - 7 4}$ & $\mathbf{7 5 +}$ \\
\hline \multicolumn{1}{c}{ Alle Rentner } & 56,5 & 31,6 & 21,5 & 11,9 \\
Paare & 62,2 & 39,3 & 29,7 & 19,4 \\
Alleinstehende Männer & 57,1 & 26,9 & 26,8 & 10,9 \\
Alleinstehende Frauen & 40,6 & 20,6 & 14,7 & 8,7 \\
\hline
\end{tabular}

Anmerkungen: Anteil der Haushalte mit positiven Arbeitseinkünften im Jahr 1993 (auch Nebentätigkeiten und gelegentliche Tätigkeiten). Nukleus-Haushalte sind hier Haushalte, die maximal aus Bezugsperson, Partner und minderjährigen Kindern bestehen. Quelle: Eigene Berechnungen auf Basis der EVS 1993

Tabelle 2 verdeutlicht, daß der Übergang von der Erwerbstätigkeit in den Ruhestand allmählich verläuft. Hierbei wurden Mehr-Generationen-Haushalte ausgeschlossen, um zu vermeiden, daß die Ergebnisse durch das Vorhandensein von erwachsenen, erwerbstätigen Kindern verfälscht werden. Noch in der Altersgruppe der 70- bis 74-Jährigen spielt Erwerbstätigkeit eine nicht unerhebliche Rolle, da etwa ein Viertel der alleinstehenden Männer dieser Altersgruppe und 30 Prozent der Zwei-Personen-Rentnerhaushalte einer Beschäftigung nachgehen. Dies spiegelt sich ebenfalls in Niveau und Zusammensetzung des Haushaltseinkommens wider, dem wir uns im folgenden Abschnitt zuwenden. 


\section{Die Einkommenssituation im Ruhestand}

In diesem Kapitel untersuchen wir Niveau, Zusammensetzung und Verteilung des Einkommens von Rentnerhaushalten auf Basis der Einkommens- und Verbrauchsstichprobe 1993 (EVS). Als Rentnerhaushalt wird ein Haushalt definiert, in dem eine Person lebt, die mindestens 65 Jahre alt ist oder mindestens 60 Jahre alt ist und ihren überwiegenden Lebensunterhalt nicht aus eigener Erwerbstätigkeit bestreitet. Komposithaushalte - Haushalte in denen neben dem Rentner und dessen Ehepartner noch andere Personen, z.B. Eltern oder erwachsene Kinder leben - werden aus der Analyse ausgeschlossen. Dies betrifft 18\% der Rentnerehepaare und jeweils $13 \%$ der alleinlebenden Männer und Frauen. Die Arbeitsstichprobe besteht dann aus 6635 Haushalten, wobei alle Ergebnisse mit den Hochrechnungsfaktoren der EVS gewichtet wurden.

Als Einkommensgröße wird das verfügbare Einkommen betrachtet, das sich aus der Summe von Arbeitseinkommen, Einkünften aus Kapitalvermögen, öffentlichen und privaten Transfers abzüglich den Beiträgen zur Sozialversicherung und direkten Steuern zusammensetzt ${ }^{6}$. Bei der Interpretation ist zu beachten, daß in der EVS zum einen Personen, die in Institutionen (z.B. Altenheime) leben, und zum anderen Haushalte mit einem Jahreseinkommen von mehr als 300.000 DM fehlen. Ersteres könnte vor allem bei der Betrachtung der ältesten Haushalte ein Problem sein, da in Deutschland etwa 10\% der über 80-Jährigen in Institutionen leben (Bickel und Jaeger, 1986). Das Fehlen der einkommensreichsten Haushalte läßt sich in Verteilungsanalysen durch Anpassung der Quintilsgrenzen berücksichtigen. Mittelwerte lassen sich aber ohne weitgehende Annahmen nicht korrigieren.

Werden in einer Tabelle Ergebnisse für Haushalte mit verschiedenen Haushaltsgrößen gezeigt, wird das verfügbare Haushaltseinkommen über die Verwendung einer Äquivalenzskala vergleichbar gemacht. Es wird die ältere OECD-Skala verwendet, die jedes zusätzliche Haushaltsmitglied über 14 Jahren mit 0,7 und jedes Kind mit 0,5 gewichtet.

\section{Niveau der verfügbaren Einkommen}

Die Mittelwerte des verfügbaren Einkommens gegliedert nach drei verschiedenen Haushaltstypen und Altersklassen gehen aus Tabelle 3 hervor. Die relative Einkommensposition

\footnotetext{
${ }^{6}$ Eine ausführliche Beschreibung der EVS 1993 und unserer Variablendefinitionen findet sich in Börsch-Supan, Reil-Held, Rodepeter, Schnabel, Winter 1999.
} 
der Rentnerhaushalte im Vergleich zu den jüngeren Kohorten wird in der Zeile „Anteil“ dargestellt und errechnet sich als Quotient des Mittelwertes der jeweiligen Rentnergruppe und dem äquivalisierten Durchschnittseinkommen der Nicht-Rentner-Bevölkerung des selben Jahres.

Das durchschnittliche Einkommen aller Rentner beträgt 88 Prozent des Äquivalenzeinkommens der jüngeren Bevölkerung und liegt damit relativ hoch. Während die alleinstehenden Rentner und Ehepaare mit 101 Prozent respektive 92 Prozent relativ gut ausgestattet sind, verfügen die alleinstehenden Rentnerinnen dagegen mit etwa 80 Prozent des Einkommens der erwerbsfähigen Bevölkerung über die niedrigsten Einkommen.

Tabelle 3: Durchschnittliches verfügbares Einkommen nach Alter und Haushaltstyp

\begin{tabular}{|c|c|c|c|c|c|}
\hline & $\begin{array}{l}\text { 60-64, } \\
\text { in Rente }\end{array}$ & $65-69$ & $70-74$ & $75+$ & $\begin{array}{c}\text { Alle über 60, } \\
\text { in Rente }\end{array}$ \\
\hline \multicolumn{6}{|l|}{ Ehepaare } \\
\hline Mittelwert (absolut) & 47,945 & 52,188 & 47,394 & 46,465 & 48,787 \\
\hline Mittelwert (äquiv.) & 27,804 & 30,346 & 27,862 & 27,332 & 28,474 \\
\hline Anteil 1 & $(0.90)$ & $(0.98)$ & $(0.89)$ & $(0.88)$ & $(0.92)$ \\
\hline Anteil 2 & {$[0.74]$} & {$[0.80]$} & {$[0.73]$} & {$[0.72]$} & {$[0.75]$} \\
\hline Fallzahlen & 1,682 & 1,766 & 1,246 & 802 & 5,496 \\
\hline \multicolumn{6}{|l|}{ Alleinstehende Männer } \\
\hline Mittelwert (absolut) & 26,464 & 33,589 & 33,714 & 30,947 & 31,775 \\
\hline Anteil 1 & $(0.85)$ & $(1.08)$ & $(1.08)$ & $(0.99)$ & $(1.02)$ \\
\hline Anteil 2 & {$[0.69]$} & {$[0.88]$} & {$[0.88]$} & {$[0.81]$} & {$[0.83]$} \\
\hline Fallzahlen & 104 & 215 & 199 & 248 & 766 \\
\hline \multicolumn{6}{|l|}{ Alleinstehende Frauen } \\
\hline Mittelwert & 25,407 & 26,220 & 26,255 & 22,950 & 25,014 \\
\hline Anteil 1 & $(0.81)$ & $(0.84)$ & $(0.84)$ & $(0.74)$ & $(0.80)$ \\
\hline Anteil 2 & {$[0.67]$} & [0.69] & [0.69] & {$[0.60]$} & {$[0.66]$} \\
\hline Fallzahlen & 516 & 882 & 946 & 777 & 3,121 \\
\hline \multicolumn{6}{|l|}{ Alle } \\
\hline Mittelwert & & & & & 26,450 \\
\hline Anteil 1 & & & & & $(0.88)$ \\
\hline Anteil 2 & & & & & {$[0.72]$} \\
\hline Fallzahlen & & & & & 9,383 \\
\hline
\end{tabular}

Anmerkungen: Einkommen in DM pro Jahr. „Anteil“ ist der Quotienten aus dem durchschnittlichen verfügbaren Äquivalenzeinkommen der jeweiligen Gruppe und dem durchschnittlichen verfügbaren Äquivalenzeinkommen der Nicht-Rentner (DM 31,217). Für Anteil 1 in runden Klammern wird die ältere OECD Äquivalenzskala (1.0, $0.7,0.5)$ benutzt; für Anteil 2 in eckigen Klammern wird alternativ eine steilere Skala benutzt $(1.0,0.5,0.3)$. Quelle: Börsch-Supan, Reil-Held, Schnabel 1998, auf Basis der EVS 1993. Gesamtdeutschland. 
Bei der Interpretation von Tabelle 3 ist zu berücksichtigen, daß die Anteile keine Ersatzquote im Lebenszyklus darstellen, da hier die Einkommen verschiedener Kohorten verglichen werden. Die Folgerung, daß die Einkommen der Rentner im Laufe ihres Lebenszyklus sinken, wäre falsch. Durch den enormen Anstieg der realen Einkommen in den fünfziger bis siebziger Jahren liegt die effektive Ersatzquote, definiert als Quotient von Einkommen im Ruhestand und Einkommen in der Erwerbsphase einer Kohorte, wesentlich höher.

Die im Vergleich zur Altersklasse 65-69 niedrigeren Einkommen der oberen Altersklassen sind einzig und allein auf die niedrigeren Erwerbseinkünfte in den oberen Altersklassen zurückzuführen. Die vergleichsweise niedrigen Einkommen der jüngsten Rentner (Altersklasse 60-64) dagegen, lassen sich durch einen Selektionseffekt erklären

Tabelle 3 zeigt außerdem die Sensitivität der Ergebnisse bezüglich der Wahl der Äquivalenzskala. Die zweite, alternativ verwendete Skala ordnet zusätzlichen Haushaltsmitgliedern ein geringeres Gewicht zu. Dies läßt jüngere Haushalte, die in der Regel größer sind, reicher und die Einkommen der Rentner niedriger erscheinen.

\section{Zusammensetzung der Einkommen}

Eine Betrachtung der Einkommenskomponenten zeigt, daß die relativ hohen Einkommen der Rentnerhaushalte vor allem auf die gesetzlichen Renten zurückzuführen sind, die das Einkommenspaket im Ruhestand in Deutschland dominieren (siehe Tabelle 4). Dies gilt insbesondere für die alleinstehenden Rentnerinnen, bei denen der Anteil der gesetzlichen Renten etwa 82 Prozent beträgt. Eine Betrachtung nach Einkommensquintilen zeigt, daß selbst im obersten Quintil noch 52 Prozent des verfügbaren Einkommens aus öffentlichen Transfers bestehen (Börsch-Supan, Reil-Held und Schnabel 1998). Vermögenseinkommen machen im Mittel je nach Haushaltstyp und Altersklasse zwischen 8 und 13 Prozent aus. Vermögenseinkommen stellen somit im höheren Alter nach dem öffentlichen Transfereinkommen die wichtigste Einkommensquelle dar. Private Transfers (vor allem aus Werksrenten) sind mit einem Anteil von 4 bis 6 Prozent relativ unbedeutend. Eine noch kleinere Rolle spielen im Durchschnitt der Rentnerhaushalte einmalige Transfers wie Erbschaften oder Auszahlungen aus Lebensversicherungen. 
Tabelle 4: Zusammensetzung des Einkommens nach Alter und Haushaltstyp

\begin{tabular}{lccccc}
\hline & $\mathbf{6 0 - 6 4}$ & $\mathbf{6 5 - 6 9}$ & $\mathbf{7 0 - 7 4}$ & $\mathbf{7 5 +}$ & alle 60+ \\
\hline Ehepaare & & & & & \\
Erwerbseinkommen & $26.7 \%$ & 15.1 & 6.4 & 3.1 & $14.7 \%$ \\
Eink. aus Vermögen & 7.3 & 10.8 & 12.4 & 12.2 & 9.4 \\
Öffentl.Transfers & 58.1 & 64.8 & 71.8 & 75.5 & 66.1 \\
Private Transfers & 3.2 & 5.0 & 5.5 & 5.2 & 4.6 \\
Unregelmäßige Eink. & 4.4 & 4.4 & 3.8 & 4.1 & 4.2 \\
\hline Alleinstehende Männer & & & & & \\
Erwerbseinkommen & $2.4 \%$ & 7.2 & 4.9 & 2.5 & $4.1 \%$ \\
Eink. aus Vermögen & 6.8 & 11.9 & 10.8 & 12.9 & 11.6 \\
Öffentl.Transfers & 80.6 & 71.3 & 77.0 & 74.3 & 75.1 \\
Private Transfers & 5,6 & 6.7 & 5.2 & 6.6 & 6.1 \\
Unregelmäßige Eink. & 3.5 & 2.9 & 2.2 & 3.7 & 3.1 \\
\hline Alleinstehende Frauen & & & & & 2.9 \\
Erwerbseinkommen & $3.7 \%$ & 3.6 & 1.9 & 0.1 & $2.8 \%$ \\
Eink. aus Vermögen & 8.2 & 7.7 & 10.2 & 8.0 & 8.3 \\
Öffentl.Transfers & 80.9 & 81.8 & 80.6 & 84.9 & 82.2 \\
Private Transfers & 3.8 & 4.2 & 4.9 & 4.5 & 4.5 \\
Unregelmäßige Eink. & 3.6 & 2.8 & 2.4 & 2.0 & 2.5 \\
\hline Quelle: Eigene Berechnungen & Bas & & & \\
\hline
\end{tabular}

Quelle: Eigene Berechnungen auf Basis der EVS 1993. Gesamtdeutschland.

Erwerbseinkommen spielen gerade bei den jüngeren Rentnern eine große Rolle. Bei den Ehepaaren im Alter von 65 bis 69 Jahren sind Erwerbseinkommen mit einem Anteil von 15 Prozent am gesamten Einkommen die zweit wichtigste Einkommensquelle, obwohl nahezu alle Bezugspersonen in Rente sind. Weiterhin läßt sich ein starker Zusammenhang zwischen dem Anteil des Arbeitseinkommens und der Höhe des gesamten Einkommens feststellen. Im obersten Quintil betragen Einkünfte aus Erwerbstätigkeit nahezu 20 Prozent des Einkommens der Rentnerhaushalte und Vermögenseinkommen weitere 16 Prozent (Börsch-Supan, Reil-Held und Schnabel 1998). In den unteren beiden Quintilen tragen die Erwerbseinkommen dagegen weniger als 5 Prozent zum verfügbaren Einkommen bei. In diesem Einkommenssegment ist die Dominanz der gesetzlichen Renten mit einem Anteil von nahezu 90 Prozent am größten. 


\section{Vermögen}

Die Beschränkung auf die Einkommenslage ergibt ein unvollständiges Bild der wirtschaftlichen Lage im Alter, denn das Vermögen spielt eine wichtige Rolle für die Bestimmung der Konsummöglichkeiten. ${ }^{7}$ Der bedeutendste Vermögensgegenstand ist das Immobilienvermögen, zumeist in der Gestalt von selbstgenutztem Wohneigentum (Schnabel 1998). Da für ältere Eigentümerhaushalte die direkten Kosten der Wohnung wesentlich niedriger liegen als für Mieterhaushalte, ist der Eigentümerstatus für die Beurteilung der Einkommenssituation älterer Haushalte von zentraler Bedeutung. Darüber hinaus können Vermögensbestände auch veräuBert werden, um den Konsum zu finanzieren.

Die Mietausgaben (einschließlich Nebenkosten) betragen für westdeutsche Haushalte in 1993 im Durchschnitt knapp 19 Prozent des verfügbaren Einkommens. Bei älteren Haushalten ist der Mietanteil am verfügbaren Einkommen mit 21 bis 23 Prozent höher als bei jüngeren Haushalten, die nur etwa 16 bis 18 Prozent ihres Einkommens für Mietzahlungen aufwenden. Ein-Personen-Haushalte weisen erwartungsgemäß eine höhere Belastung auf. So geben ältere alleinstehende Frauen etwa 25 Prozent ihres Einkommens für Mietzahlungen aus. ${ }^{8}$ Während die durchschnittliche Miete etwa 6000 DM pro Jahr beträgt, wenden Eigentümerhaushalte im Durchschnitt nur 3200 DM für ihre Wohnung oder ihr Haus an auf. Hierbei schließen die Eigentümerkosten Kreditverpflichtungen, Instandsetzungs- und Erhaltungsaufwand ein. Für die Eigentümerhaushalte ohne Restschuld betragen die Ausgaben im Jahr sogar nur etwa 1800 DM (Börsch-Supan, Reil-Held und Schnabel 1998). Die wirtschaftliche Lage von Eigentümerhaushalten ist daher - selbst bei gleichen verfügbaren Einkommen - schon von der Kostenseite her günstiger als die von Mieterhaushalten. Zusätzlich haben Eigentümerhaushalte im Mittel deutlich höhere laufende Einkommen und ein vielfach höheres Vermögen (Schnabel 1998). Die Verteilung des Immobilienvermögens auf die Haushaltstypen ist nicht homogen. Während etwa 52 Prozent der Rentner-Ehepaare in Eigentum wohnen, sind es unter den alleinstehenden Männern nur etwa 40 Prozent und unter den alleinstehenden Rentnerinnen nur gut 30 Prozent.

\footnotetext{
${ }^{7}$ Vermögen kann auf verschiedene Weisen für den Konsum genutzt werden. Zum einen kann Vermögen für die Finanzierung von Konsum aufgelöst werden. Zum anderen spendet selbstgenutztes Wohneigentum unmittelbaren Konsum.

${ }^{8}$ Quelle: Eigene Berechnungen auf Basis der EVS 1993
} 


\section{Verteilung der verfügbaren Einkommen}

Nachdem bislang nur die Einkommensunterschiede in den Mittelwerten zwischen den einzelnen Haushaltstypen betrachtet wurden, soll nun näher auf die Verteilung eingegangen werden. Hierbei soll auch der Frage nach eventuell vorhandenen Armutslücken im Alter nachgegangen werden. Als Maß der Einkommensverteilung zeigt Tabelle 5 den Abstand zwischen dem obersten und dem untersten Einkommensdezil (als Quotient der Dezilsgrenzen).

Tabelle 5: Verteilung des verfügbaren Einkommens nach Alter und Haushaltstyp

\begin{tabular}{|c|c|c|c|c|c|}
\hline & $\begin{array}{c}\text { 60-64, } \\
\text { in Rente }\end{array}$ & $65-69$ & $70-74$ & über75 & $\begin{array}{l}\text { Alle 60+, } \\
\text { in Rente }\end{array}$ \\
\hline \multicolumn{6}{|l|}{ Ehepaare } \\
\hline Median & 41,539 & 43,727 & 38,969 & 39,597 & 41,193 \\
\hline 90stes Dezil & 81,050 & 94,001 & 84,070 & 82,043 & 85,841 \\
\hline 10tes Dezil & 26,738 & 26,977 & 25,579 & 23,268 & 26,020 \\
\hline $90 / 10$ & 3.03 & 3.48 & 3.29 & 3.53 & 3.30 \\
\hline \multicolumn{6}{|c|}{ Alleinstehende Männer } \\
\hline Median & 23,260 & 28,925 & 29,358 & 25,976 & 27,231 \\
\hline 90stesDezil & 43,437 & 58,646 & 62,575 & 59,149 & 57,131 \\
\hline 10tes Dezil & 12,231 & 17,132 & 18,472 & 12,938 & 14,168 \\
\hline 90/10 & 3.55 & 3.42 & 3.39 & 4.57 & 4.03 \\
\hline \multicolumn{6}{|c|}{ Alleinstehende Frauen } \\
\hline Median & 22,134 & 23,297 & 23,441 & 21,326 & 22,367 \\
\hline 90stes Dezil & 43,322 & 44,206 & 42,589 & 36,665 & 41,020 \\
\hline 10tes Dezil & 13,667 & 15,044 & 14,363 & 13,554 & 14,033 \\
\hline $90 / 10$ & 3.17 & 2.94 & 2.97 & 2.71 & 2.92 \\
\hline
\end{tabular}

Anmerkungen: Verfügbares Einkommen in DM pro Jahr, nicht äquivalisiert. Quelle: Börsch-Supan, ReilHeld, Schnabel 1998, auf Basis der EVS 1993, Gesamtdeutschland.

Die Einkommensungleichheit erscheint im internationalen Vergleich als relativ gering (siehe Hauser et al., 1997 und Disney et.al., 1998). Zwischen den alleinlebenden Rentnern ist die Ungleichheit am größten. Die oberen 10 Prozent der Einkommensverteilung verfügen in dieser Bevölkerungsgruppe über ein etwa viermal so hohes Einkommen als die unteren 10 Prozent. Über die Altersklassen hinweg läßt sich keine systematische Entwicklung der Ungleichheit feststellen.

Eine Betrachtung nach Einkommensdezilen über die gesamte Bevölkerung bestätigt die Heterogenität der Rentnerhaushalte. Der Anteil der älteren Haushalte in den obersten Einkommensdezilen nimmt kontinuierlich ab, wobei die alleinstehenden Männer in den oberen Dezilen noch stark repräsentiert sind, während die alleinlebenden Rentnerinnen überproportional 
im unteren Bereich der Verteilung wiederzufinden sind. Ihr Anteil am untersten Dezil beträgt etwa 13\%, im obersten Dezil dagegen nur etwa 4\% (Börsch-Supan, Reil-Held und Schnabel 1998).

Wie schon mehrfach gezeigt, ist unter den Rentnerhaushalten die Einkommenslage von alleinstehenden Frauen am schlechtesten. Ein ähnliches Bild ergibt sich bei der Analyse des Sozialhilfebezugs. Etwa 3 Prozent der alleinstehenden Frauen der EVS 1993 beziehen Sozialhilfe (gegenüber 3,5 Prozent in der Gesamtbevölkerung). Dies bestätigt, daß Armut kein spezifisches Problem von Rentnern oder Rentnerinnen im besonderen ist. Dieses Ergebnis bestätigt auch andere Untersuchungen, die eine Abnahme der Altersarmut in den 80er Jahren feststellen (z.B. Becker 1997, Motel 1998).

Die Zahl der Sozialhilfebezieher stellt wegen des Problems der Nichtbeanspruchung eine Untergrenze für die Bedürftigkeit dar. Eine Auswertung der EVS 1993, bei der wir den Bedarf nach dem Sozialhilfegesetz schätzen, ergibt, daß etwa 1,5 bis 3 Prozent der älteren Frauen einen Anspruch haben, der nicht geltend gemacht wird. ${ }^{9}$ Wir berichten mit Absicht keine Punktschätzung, da die Schätzungen aus mehreren Gründen nicht robust sind. Zum einen konzentrieren sich viele Haushalte in der Nähe der Einkommensgrenze. Zum anderen gibt es bei der Sozialhilfegewährung einen Ermessensspielraum, den wir nicht modellieren können - etwa bei der Übernahme der Mietausgaben oder der Anerkennung eines Mehrbedarfs. Werden Sozialhilfebezug und Nichtinanspruchnahme zusammengerechnet, dann sind nach unseren Schätzungen zwischen 4,5 und 6 Prozent der älteren, alleinstehenden Frauen bedürftig.

\section{Ausblick: Erwerbsbeteiligung, Rentenreformen und Einkommen im Ru- hestand}

\section{Entwicklung von Erwerbsbeteiligung und Rentenanwartschaften von Frauen}

Die Absicherung von Frauen im Alter hat sich in den letzten Jahrzehnten deutlich verbessert sowohl absolut als auch relativ zur Absicherung der Männer. Insbesondere das Rentenreformgesetz von 1972 bewirkte einen deutlichen Anstieg der Renten von Frauen (so etwa durch Einführung von Zuschlägen für Pflichtversicherungszeiten mit niedrigen Verdiensten); in der Folge gab es weitere Reformen, die beispielsweise die Anrechnung von Kindererziehungszei- 
ten verbesserten. Zum anderen hat sich aber auch die Zahl der Beitragszeiten von Frauen durch die verstärkte Erwerbsbeteiligung erhöht: Im Rentenzugang nahm von 1972 bis 1996 die mittlere Zahl der Versicherungsjahre um 18 Prozent und die mittlere Zahl der Entgeltpunkte sogar um 36 Prozent zu. Im Ergebnis sind die eigenen Renten von Frauen von 1972 bis 1996 um 88 Prozent gestiegen, während gleichzeitig die Renten von Männern nur um 28 Prozent und die Nettoentgelte sogar nur um 15 Prozent gestiegen sind. Die Verbesserung der Lage von älteren Frauen läßt sich ebenfalls ablesen am Rückgang der Zahl der Sozialhilfeempfängerinnen. Im Jahr 1993 bezogen nach unseren Berechnungen auf Basis der EVS nur noch 3 Prozent der älteren, alleinstehenden Frauen Sozialhilfe.

Die weitere Zunahme der Versicherungszeiten von Frauen und damit eine Verbesserung der Lage von Rentnerinnen ist bereits heute absehbar. Dies geht aus einer Analyse mit dem SOEP der Jahre 1985 bis 1996 hervor. Hierfür wurden aus dem Lebenslauf- und dem Erwerbskalendarium die Zeiten der Erwerbstätigkeit ermittelt und daraus die Gesamtzahl der Erwerbszeiten geschätzt. $^{10}$ So weisen die 55- bis 59-Jährigen Frauen des Jahres 198517 Erwerbsjahre auf. Diese Frauen sind etwa um das Jahr 1990 in Rente gegangen. Schon 11 Jahre später, im Jahr 1996, weisen die 55- bis 59-Jährigen Frauen bereits 22 Erwerbsjahre auf. Diese Kohorte, die um die Jahrtausendwende in Rente geht, hat schon heute etwa 30 Prozent höhere Rentenanwartschaften gebildet als die um 10 Jahre ältere Kohorte. Die folgenden Kohorten werden nochmals höhere Rentenanwartschaften gebildet haben, denn die Kohorte der 50- bis 54Jährigen hatte bereits 1996 mehr Erwerbsjahre angesammelt als die vorhergehende Kohorte. Das Problem der Altersarmut von Frauen aufgrund fehlender eigener Renten wird demnach noch weiter in den Hintergrund treten.

\section{Demographische Entwicklung und Rentenreformen}

Die absehbare demographische Entwicklung läßt jedoch eine Verschlechterung der sozialen Lage von Rentnern befürchten. Als eine erste Reaktion auf die demographische Entwicklung hat das Rentenreformgesetz 1992 mit der Einführung von Abschlägen bei vorzeitiger Verren-

\footnotetext{
${ }^{9}$ Der nicht geltend gemachte Bedarf beträgt im Mittel 140 DM pro Monat, der Median 90 DM. Dies ist wesentlich weniger als die Leistungen in Höhe von 260 DM (resp. 190 DM), die die Sozialhilfebezieher erhalten.

${ }^{10}$ Zeiten der Teilzeitbeschäftigung und der beruflichen Ausbildung wurden hierbei nur zur Hälfte gezählt. Die Ergebnisse des Kohortenvergleichs sind jedoch nicht sensitiv bezüglich der spezifischen Gewichtung.
} 
tung eine wesentliche Änderung gebracht. ${ }^{11}$ Für Personen, die mit 60 in Rente gehen, wird sich das relative Rentenniveau um 18 Prozent vermindern. Im Bereich der unteren Einkommen scheint dies einen schmerzlichen Einschnitt darzustellen. Dieser Abschlag läßt sich jedoch vermeiden, sofern die Rente erst nach dem 65 . Geburtstag beansprucht wird. ${ }^{12}$

Die weitere Entwicklung der Einkommenslage von Rentnern hängt zum einen von künftigen politischen Entscheidungen (in erster Linie von der Ausgestaltung der kommenden Rentenreformen), aber auch ganz entscheidend von den Verhaltensreaktionen der Individuen ab. Hier sind in erster Linie die Erwerbsbeteiligung und das Sparverhalten im Lebenszyklus zu nennen. Einige Tendenzen lassen sich bereits erkennen. So kann es als sicher gelten, daß die Nettoersatzquote in den nächsten Jahren zumindest auf das Niveau der sechziger Jahre - etwa 64 Prozent - zurückgeführt wird. ${ }^{13}$ Dies würde geringere Renten relativ zu den Erwerbseinkommen bedeuten, sofern Erwerbsbeteiligung und Rentenzugang konstant bleiben. Dies ist keineswegs gleichbedeutend mit einem absoluten Rückgang der Renten.

In der hypothetischen Situation einer sofortigen, zehnprozentigen Kürzung der öffentlichen Renten würden die Konsummöglichkeiten von Rentnerhaushalten im unteren Bereich der Einkommensverteilung erheblich eingeschränkt. ${ }^{14}$ Die Zahl der sozialhilfeberechtigten Rentnerinnen würde nach unseren Berechnungen um 60 Prozent höher liegen, was einem Anteil an den alleinstehenden Rentnerinnen von etwa 8 bis 10 Prozent entspräche (Börsch-Supan, ReilHeld und Schnabel 1998). Diese starke Zunahme ist darauf zurückzuführen, daß der Einkommensunterschied zwischen dem 5-Prozent und dem 10 Prozent Perzentil sehr gering ist. Diese hypothetische Situation ist allerdings aus mehreren Gründen unrealistisch. Erstens, würde eine Senkung der Ersatzquote langfristig erfolgen; daher bliebe reichlich Zeit, um eine etwaige

\footnotetext{
${ }^{11}$ Weitere wichtige Änderungen waren etwa die Umstellung von der brutto- auf die nettolohnbezogene Rentenanpassung und die Kürzung der Anrechnungszeiten für Berufsausbildung. Die Kürzung der Anrechnung von Ausbildungszeiten betrifft Personen mit langer Ausbildung, also vorwiegend gut verdienende Akademiker.

${ }^{12}$ Für ökonometrische Analysen, die den Einfluß der Rentenreform auf das Verrentungsalter bestimmen, siehe Börsch-Supan (1992), Schmidt (1995), Siddiqui (1997) und Börsch-Supan (1998b).

${ }^{13}$ Das noch unter der alten Bundesregierung verabschiedete, nun aber aufgeschobene, Rentenreformgesetz 1999 sah eine Senkung der Nettoersatzquote auf 64 Prozent vor. Dies entspräche einer Minderung des relativen Rentenniveaus um 10 Prozent.

${ }^{14}$ Im Bereich der Mittelschicht werden heute die Ruhestandseinkommen nicht vollständige konsumiert. Statt dessen wird ein beträchtlicher Teil gespart und/oder zeit Lebens transferiert. Die erheblichen Vermögensbestände werden in der Ruhestandsphase schon gar nicht angetastet. Eine zehn-prozentige Kürzung der öffentlichen Renten würde daher den Lebensstandard der Rentner in der Mittelschicht nicht beeinträchtigen. Anders sieht dies im Bereich des unteren Viertels der Einkommensverteilung und ganz besonders im unteren Zehntel aus. Hier können Rentenkürzungen nicht von heute auf morgen ohne Folgen für den Konsum bleiben.
} 
Rentenlücke durch private Vorsorge zu schließen, die aus den ersparten Beiträgen zur gesetzlichen Rentenversicherung gespeist werden kann. Zweitens, hat sich in der jüngeren Vergangenheit die Erwerbsbeteiligung von Frauen erhöht; dies führt zu höheren eigenen Rentenanwartschaften der kommenden Generationen von Rentnerinnen. Schließlich kann ein Verzicht auf Frühverrentung entscheidend zur Erhöhung der Rentenanwartschaften beitragen. Diese Mechanismen können die Folgen einer niedrigeren Ersatzquote des Umlageverfahrens zumindest teilweise kompensieren und damit zu einer Stabilisierung der effektiven Ersatzquote beitragen. 


\section{Literaturverzeichnis}

Becker, I., 1997, Die Entwicklung von Einkommensverteilung und Einkommensarmut in den alten Bundesländern von 1962 bis 1988. In: Becker, I. und R. Hauser (Hg.): Einkommensverteilung und Armut. Deutschland auf dem Weg zur VierfünftelGesellschaft? Campus Verlag, Frankfurt, New York.

Bickel, H. und J. Jaeger, 1986, Die Inanspruchnahme von Heimen im Alter, Zeitschrift für Gerontologie 19: 30-39.

Börsch-Supan, A. ,1992, Population Aging, Social Security Design, and Early Retirement, Journal of Institutional and Theoretical Economics 148.

Börsch-Supan, A. (1998): Incentive Effects of Social Security on Labor Force Participation: Evidence in Germany and across Europe, forthcoming in: Journal of Public Ecomomics, 1999.

Börsch-Supan, A., A. Reil-Held, R. Rodepeter, R. Schnabel und J. Winter, 1999, Ersparnisbildung in Deutschland: Meßkonzepte auf Basis der EVS. SFB Arbeitspapier Nr. 99-02, Universität Mannheim.

Börsch-Supan, A, Reil-Held, A. und R. Schnabel, 1998, Pension Provision in Germany. SFB Arbeitspapier Nr. 98-07, Universität Mannheim.

Börsch-Supan, A. und R. Schnabel, 1998a, Social Security and Declining Labor Force Participation in Germany, American Economic Review 88.2, 173-178.

Börsch-Supan, A. und R. Schnabel, 1998b, Social Security and Retirement in Germany, in: J. Gruber and D.A. Wise (eds), Social Security and Retirement around the World, University of Chicago Press: Chicago, 135-180.

Disney, R., M. Mira d'Ercole und P. Scherer, 1998, Resources in Retirement, OECD Working paper AWP 4.3, Paris.

Hauser, Richard, K. Mörsdorf und F. Tibitanzl, 1997, Dokumentation empirischer Daten zur Alterssicherung in der Europäischen Union und Nordamerika, mimeo, Universität Frankfurt.

Motel, A., 1998, Einkommen und Vermögen älterer Menschen, subjektive Bewertungen des persönlichen Lebensstandards und Erwartungen seiner künftigen Entwicklung. In: M. Kohli und H. Künemund (Hg.): Die zweite Lebenshälfte - Gesellschaftliche Lage und Partizipation. Forschungsgruppe Altern und Lebenslauf, Freie Universität Berlin.

Schmidt, P., 1995, Die Wahl des Rentenalters - Theoretische und empirische Analyse des Rentenzugangsverhaltens in West- und Ostdeutschland. Lang, Frankfurt.

Schnabel, R., 1998, Vermögen und Ersparnis im Lebenszyklus in Westdeutschland, Arbeitspapier, Universität Mannheim, 1998.

Siddiqui, S., 1997, The Pension Incentive to retire: Empirical Evidence for West Germany, Journal of Population Economics 10(4): 463-86.

VDR, Verband deutscher Rentenversicherungsträger, 1997, Die Rentenversicherung in Zeitreihen, Frankfurt am Main: VDR.

VDR, Verband Deutscher Rentenversicherungsträger, 1997, Rentenzugang 1995, Frankfurt am Main: VDR. 